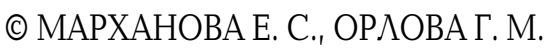

УАК 616.124.2-007.61-06:616.12-008.331.1(571.54)

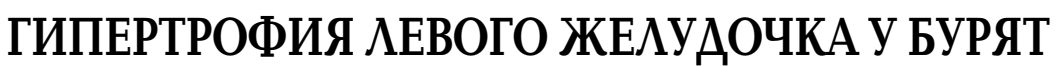 С АРТЕРИАЛЬНОЙ ГИПЕРТОНИЕЙ
}

\author{
Е. С. Марханова, Г. М. Орлова
}

ГБОУ ВПО Иркутский государственный медицинский университет Министерства здравоохранения РФ, ректор - А. м. н., проф. И. В. Малов; кафедра госпитальной терапии, зав. - А. м. н., проф. Г. М. Орлова.

\begin{abstract}
Цель исслеgования. Изучить частоту гипертрофии левого желуgочка, особенности ремоgелирования миокарgа левого желуgочка у пациентов бурятской национальности с артериальной гипертонией.

Материалы и метоgы. В ислеgование включено 100 пациентов бурятской национальности, из них 47 мужчин и 53 женщины. Результаты. Распространенность гипертрофии левого желуgочка среgи бурятских пациентов с артериальной гипертонией составляет $62 \pm 4,9 \%$. Наиболее частым типом ремоелирования левого желуgочка у бурятских пациентов является концентрическая гипертрофия левого желудочка. Выявляется тенденция к более высокой частоте и тяжести гипертрофии левого желудочка у женщин.
\end{abstract}

Заключение. Выявлена высокая распространенность и генgерные отличия гипертрофии левого желуgочка у бурят. Ключевые слова: гипертрофия левого желуgочка, буряты, артериальная гипертония.

\section{LEFT VENTRICULAR HYPERTROPHY IN BURYATS WITH ARTERIAL HYPERTENSIVE}

\author{
E. S. Markhanova, G. M. Orlova \\ Irkutsk State Medical University, Russia
}

The aim of the research. To study features of left ventricular hypertrophy in Buryats with arterial hypertensive.

Materials and methods. The study included 100 patients of Buryat nationality, 47 men and 53 women.

The Results. The prevalence of left ventricular hypertrophy among the Buryat patients with hypertensive was $62 \pm 4,9 \%$. The most common type of left ventricular remodeling in Buryat patients is concentric left ventricular hypertrophy. It is revealed a trend towards higher frequency and severity of left ventricular hypertrophy in women.

Conclusion. It was found high prevalence and gender differences of left ventricular hypertrophy in Buryats.

Key words: left ventricular hypertrophy, Buryats, arterial hypertension.

Исследования последних лет позволили прийти к вывоАу о том, что гипертрофия левого желудочка сердца (ГАЖ) является независимым фактором риска инфаркта миокарда, сердечной недостаточности, желудочковых нарушений ритма и внезапной смерти. Так, поданным крупномасштабного Фремингемского исследования установлено, что ГАЖ является сильнейшим индикатором быстрого развития фатального атеросклероза. Масса левого желудочка (ЛЖ) прямо коррелирует с ростом сердечно-сосудистых заболеваний и смертности [8]. В развитии ГАЖ при артериальной гипертонии (АГ), наряду с гемодинамическими, нейрогенными и гуморальными, существенную роль играют генетические факторы. J.N. Bella, H.H. Göring в своем научном обзоре обобщили данные актуальных генетических исследований и пришли к выводу о высокой наследуемости массылевого желудочка и генетической предрасположенности к развитию ГАЖ [4].

О генетической предрасположенности свидетельствуют также и этнические различия ГАЖ. Исследования частоты и особенностей ремоделирования миокарда у азиатских народов малочисленны. Отдельные работы свидетельствуют о более низкой частоте и тяжести ГАЖ у азиатских народов в сравнении европеоидами, латиноамериканцами и афроамериканцами [11].
В России, несмотря на ее многонациональный состав, подобные исследования весьма малочисленны, а их результаты во многом противоречивы. Так, согласно исследованию С.Н. Морозова (2009) частота ГАЖ у больных АГ якутской национальности ниже, чем у русских жителей Севера [2]. Поданным исследования Е.ААбрамова, тяжесть и выраженность ГАЖ у корейцев Приморского края (мигрантов в 2-3 поколении) была менее выраженной, чем у славян [1]. Исследований ГАЖ у бурятских пациентов с АГ не проводилось.

Цель исследования: изучить частоту ГАЖ, особенности ремоделирования миокарда левого желудочка у пациентов бурятской национальности с АГ.

\section{Материалы и методы}

В исследование методом случайной выборки включено 100 пациентов бурятской национальности с АГ, проходивших обследование и лечение в кардиологических стационарах Иркутской областной клинической больницы и республиканской больницы г. Улан-УАэ. Среди них 47 мужчин, средний возраст которых составил 55,4 \pm 8,1 лет, 53 женщины, средний возраст - 63,2 $\pm 11,4$ лет, р >0,05.

Аиагностика АГ осуществлялась в соответствии с российскими национальными рекомендациями по диагностике и лечению АГ (2010). По степени АГ пациенты 
распределились следующим образом: АГ 1 степени - у 12 $(12 \pm 3,2 \%)$, АГ 2 степени - у $23(23 \pm 4,2 \%)$, АГ 3 степени - у $65(65 \pm 4,8 \%)$ пациентов. У большинства пациентов $(84 \pm 3,7 \%)$ определялась АГ 3 стадии. Медианадлительности АГ составила $8[3 ; 15]$ лет. У 80 пациентов диагностирована ишемическая болезнь сердца (ИБС), АОля больных ИБС среди мужчин и женщин не различалась (40 женщин, 40 мужчин), у 36 из них диагноз ИБС верифицирован путем проведения коронароангиографии. По количеству пораженных коронарных сосудов пациенты распределились следующим образом: 1-сосудистое поражение у $13(36,1 \pm 8 \%)$ пациентов, 2-сосудистое - у $12(33,3 \pm 7,9 \%)$ пациентов, 3-сосудистое - у 11(30,6 6 7,7\%) пациентов.

Всем пациентам проведено комплексное лабораторное и инструментальное обследование. Аабораторное обслеАование включало определение липидного состава крови, уровня гликемии, креатинина крови Аля расчета скорости клубочковой фильтрации. Определение геометрии левого желудочка проводилось эхокардиографически. Масса миокарда левого желудочка (ММАЖ) рассчитывалась по формуле RB Devereux [7], при индексации по площади поверхности тела определялся индекс массы миокарда левого желудочка (ИММЛЖ), на основании которого выявлялась гипертрофия левого желудочка. Нормативные значения ИММАЖ, согласно рекомендациям по количественной оценке структуры и функции камер сердца американского эхокардиографического общества и европейской эхокарАиографиической ассоциации [3], составляют 115 г/м² Аля мужчин и 95 г $/ \mathrm{M}^{2}$ Аля женщин. Выраженность ГАЖ и тип ремоделирования определялись в соответствии с вышеупомянутыми рекомендациями.

Медикаментозную гипотензивную терапию получали 70 человек, причем в режиме монотерапии всего 13 человек, остальные получали комбинированную терапию, наиболее часто встречающиеся комбинации лекарственных препаратов: ингибиторы АПФ (или блокаторы рецепторов к ангиотензину 2) + бета-блокаторы, ингибиторы АПФ + бета-блокаторы + диуретики. Кроме того, 13ะ4,8\% пациентов принимали антагонисты кальция. Медиана длительности регулярного приема гипотензивных препаратов составила 2 [1; 5] года.

Статистический анализ осуществлен с использованием пакета программ «Statistica v. 7.0». Характер распределения признака оценивался по критерию Шапиро-Уилка. Сравнение признаков с нормальным распределением осуществлялось методом Стьюдента, с распределением, отличным от нормального, - методом Манна-Уитни. Сравнение категориальных переменных производилось с помощью критерия $\chi^{2}$. Аля выявления связи количественных показателей применен метод ранговой корреляции Спирмена. Количественные данные с нормальным распределением признака представлены в виде среднего значения со средним квадратичным отклонением $(\mathrm{M} \pm \mathrm{m})$, с, отличающимся от нормального распределением признака - в виде медианы и интерквартильного промежутка (Ме [Q25;Q75]), данные качественных признаков - в виде абсолютных значений, процентных долей и их стандартных ошибок. Статистически значимыми считали различия при $\mathrm{p}<0,05$. В проведенном исследовании соблюдены все этические нормы, больными подписано информированное согласие.

\section{Результаты и обсуждение}

Гипертрофия левого желудочка выявлена у 62 (62 $44,9 \%)$ пациентов. При этом медиана ММ ЖЖ составила 214,8 $[172,4 ; 256,6]$ г, ИММ $Ж Ж ~-~ 112,1 ~[95,5 ; ~ 137,4] ~ г / \mathrm{M}^{2}$, относительная толщина стенок $\Lambda$ (ОТС) - 0,44 [0,4; 0,49]. По выраженности ГАЖ пациенты распределились следующим

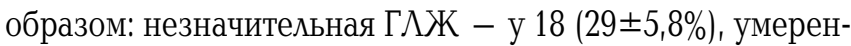
ная - у $15(24,2 \pm 5,4 \%)$, значительная - у $29(46,8 \pm 6,3 \%)$ пациентов.

По данным литературы, распространенность ГАЖ варьирует в широких пределах в зависимости от способа ее определения (ЭКГ, ЭХОКГ, магнитно-резонансная томография сердца) и индексации ММАЖ.

В масштабном исследовании, проведенном в Китае, из 4270 пациентов страдающих артериальной гипертонией, ГАЖ была выявлена в $42,7 \%$ и преобладала среди женщин (45,4\% против $37,4 \%$ у мужчин), а в возрастной группе 65 75 лет у женщин достигала 50,9\% [12]. В популяционном исследовании ГАЖ в Таиланде частота ГАЖ среди 157 пожилых тайцев старше 60 лет варьировала от 28 до 62\% [5]. Результаты уже цитируемого исследования ГАЖ у якутов, страдающих АГ, демонстрируют меньшую частоту ГАЖ: $13,3 \%$ среди мужчин и 9,3\% среди женщин, хотя в старшей возрастной группе распространенность ГАЖ была вдвое больше [2].

Возможно, выявление у бурятских пациентов с АГ существенно более высокой частоты ГАЖ объясняется включением в исследование большой доли пациентов с 3 стадией АГ, что означает наличие у них отягощающих ассоциированных клинических состояний, таких как ИБС.

Гендерные особенности частоты и выраженности ГАЖ у бурятских пациентов представлены в табл. 1.

По нашим данным, ГАЖ встречалась почти в 1,5 раза чаще у женщин, чем у мужчин, хотя различия не достигли порога достоверности. Что касается абсолютных значений

Таблица 1

\section{Гендерная характеристика частоты и выраженности гипертрофия левого желуgочка у бурятских пациентов с АГ}

\begin{tabular}{|c|c|c|c|}
\hline Показатель & $\begin{array}{c}\text { Мужчины } \\
(\mathrm{n}=47)\end{array}$ & $\begin{array}{c}\text { Женщины } \\
(\mathrm{n}=53)\end{array}$ & $\mathrm{p}$ \\
\hline Частота ГЛЖ, абс. (\%) & $25(53,2 \pm 7,3 \%)$ & $37(69,8 \pm 6,3 \%)$ & 0,09 \\
\hline $\begin{array}{c}\text { ММЛЖ, Г } \\
\text { Me [Q25; Q75] }\end{array}$ & $\begin{array}{c}283,1 \\
{[256,6 ; 334,6]}\end{array}$ & $\begin{array}{c}220,8 \\
{[194 ; 242]}\end{array}$ & $<0,001$ \\
\hline $\begin{array}{l}\text { ИММЛЖ, г/м² } \\
\text { Мe [Q25; Q75] }\end{array}$ & $\begin{array}{c}136,6 \\
{[129,1 ; 160,2]}\end{array}$ & $\begin{array}{c}126,3 \\
{[107,2 ; 146,5]}\end{array}$ & 0,009 \\
\hline $\begin{array}{c}\text { OTC } \\
\text { Me [Q25; Q75] }\end{array}$ & $\begin{array}{c}0,44 \\
{[0,39 ; 0,46]}\end{array}$ & $\begin{array}{c}0,43 \\
{[0,41 ; 0,48]}\end{array}$ & 0,7 \\
\hline $\begin{array}{c}\text { Частота значительной } \\
\text { ГЛЖ, абс. (\%) }\end{array}$ & $8(32 \pm 6,8 \%)$ & $21(56,8 \pm 6,8 \%)$ & 0,05 \\
\hline
\end{tabular}


ММАЖ, то они достоверно выше в мужской группе, это вполне закономерно, учитывая анатомические особенности сердца. Как видно из табл. 1, у мужчин ММ ЖЖ на $30 \%$ больше, чем у женщин, это же касается и ИММ $Ж$, который в среднем на 10 г/м² превышает значения у женщин. ОАнако, несмотря на это, ГАЖ в женской группе носит более выраженный характер, что можно подтвердить высокой частотой значительной ГАЖ $(56,8 \pm 6,8 \%$ по сравнению с $32 \pm 6,8 \%$ у мужчин). Таким образом, согласно данным нашего исследования, выявляется тенденция к обнаружению более высокой частоты ГАЖ у женщин, чем у мужчин. Частота значительной ГАЖ значимо выше у женщин по сравнению с мужчинами.

Анализ литературы подтверждает полученные нами данные. По данным мета-анализа, проведенного C. Cuspidi et al., который включил в себя 30 исследований за десять лет (37700 пациентов с АГ), ГАЖ у женщин выявляется значимо чаще, чем у мужчин (37,9-46,2\% против $36,0-43,5 \%$, соответственно) [6].

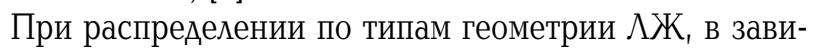
симости от ИММ АЖ и ОТС, выяснилось, что у 41 пациента определялась концентрическая ГАЖ (что составляет $41 \pm 4,9 \%$ от всей выборки и $66,1 \pm 6 \%$ от доли пациентов с ГАЖ). При этом у женщин концентрическая гипертрофия $\Lambda Ж$ регистрировалась достоверно чаще, чем у мужчин. Эксцентрическая ГАЖ, как и эксцентрическое ремоделирование $\Lambda Ж$ встречались в 2 раза реже, чем концентрическая ГАЖ и не имели различий по полу. Нормальная геометрия левого желудочка развивалась у пациентов с АГ гораздо реже (у $12 \pm 3,2 \%)$, при этом ее частота у женщин в 2 раза меньше, чем у мужчин (табл. 2).

Неблагоприятная прогностическая значимость концентрической ГАЖ была подтверждена многочисленными исследованиями. Так, по данным Фремингемского исслеАования, концентрическая гипертрофия ассоциирована с худшим прогнозом по сравнению с эксцентрической, далее следуют больные с концентрическим ремоделированием, которые также имеют большее количество осложнений, чем больные с нормальной геометрией $\Lambda Ж ~[9,10]$.

Наличие концентрической ГАЖ является самостоятельным фактором риска плохого прогноза, прежде всего

\section{Гендерная характеристика типов ремоgелирования левого желуgочка у бурятских пациентов с АГ}

\begin{tabular}{|c|c|c|c|c|}
\hline $\begin{array}{c}\text { Тип } \\
\text { ремоделирования }\end{array}$ & $\begin{array}{c}\text { Мужчины } \\
\mathrm{n}=47\end{array}$ & $\begin{array}{c}\text { Женщины } \\
\mathrm{n}=53\end{array}$ & $\begin{array}{c}\text { Всего } \\
\mathrm{n}=100\end{array}$ & $\mathrm{p}$ \\
\hline КГЛЖ, абс. (\%) & $16(34 \pm 6,9 \%)$ & $25(47,2 \pm 6,9 \%)$ & $41(41 \pm 4,9 \%)$ & 0,001 \\
\hline ЭГЛЖ, абс. (\%) & $9(19,1 \pm 5,7 \%)$ & $12(22,6 \pm 5,7 \%)$ & $21(21 \pm 4,1 \%)$ & $>0,05$ \\
\hline КРЛЖ, абс. (\%) & $14(29,8 \pm 6,7 \%)$ & $12(22,6 \pm 5,7 \%)$ & $26(26 \pm 4,4 \%)$ & $>0,05$ \\
\hline НГЛЖ, абс. (\%) & $8(17,1 \pm 5,5 \%)$ & $4(7,6 \pm 3,6 \%)$ & $12(12 \pm 3,2 \%)$ & $>0,05$ \\
\hline
\end{tabular}

Примечание: КГАЖ - концентрическая гипертрофия левого желуgочка ГАЖ, ЭГАЖ - эксцентрическая ГАЖ, КРАЖ - концентрическое ремовелирование АЖ, НГАЖ - нормальная геометрия АЖ. в плане развития ИБС и ее осложнений, поэтому высокая частота концентрической ГАЖ в нашей выборке, возможно, могла быть обусловлена большой долей пациентов с ИБС.

У $52(83,9 \pm 4,7 \%)$ больных с ГлЖ выявлена ИБС. Безусловно, наличие ИБС существенно влияет на структуру и функции миокарда левого желудочка. ОАнако, в нашем исследовании группы больных с ГАЖ и без ГАЖ не различались по доле больных ИБС: $52(83,9 \pm 4,7 \%)$ в группе с ГАЖ против $28(73,7 \pm 7,1 \%)$ в группе без ГАЖ, $\mathrm{p}>0,05$. Аоля больных ИБС среди мужчин и женщин также не имела существенных различий в обеих сравниваемых группах. Это обстоятельство позволило нам проводить дальнейший анализ без учета ИБС.

Был проведен сравнительный анализ двух групп больных: с ГАЖ и без ГАЖ по возрасту пациентов, доле курящих пациентов, различным характеристикам АГ, липидному спектру крови, показателям почечной функции, абдоминального ожирения, сопутствующей патологии.

Существенные различия между группами получены по следующим показателям:

- максимальный уровень диастолического АА (ААА макс): 100 [100;110] против 100 [90;110] мм рт. ст., р $=0,038$;

- объем фракции выброса (ФВ):60,5 [56;63] против 62,5 $[60 ; 67] \%, p=0,04$.

- уровень липопротеидов высокой плотности (АПВП): $1,4[1,18 ; 1,78]$ против $1,2[1,05 ; 1,39]$ ммоль $/ \Lambda_{1} \mathrm{p}=$ 0,017

- уровень липопротеидов низкой плотности (АПНП): $2,74[2,03 ; 3,8]$ против $3,6[2,9 ; 4,2]$ ммоль $/ \Lambda, p=0,04$;

- коэффициент атерогенности (КА): $2,4[1,65 ; 3,6]$ против $3,55[2,9 ; 4,4]$

Эти данные подтвердили ключевую роль АГ в развитии ГАЖ. Корреляционный анализ установил прямую связь

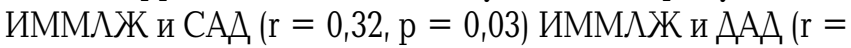

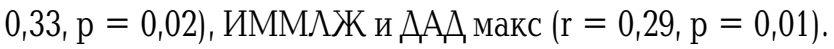

Аислипидемия не рассматривается как непосредственный предиктор ГАЖ, влияние этого фактора на ремоделирование миокарда опосредуется через развитие ИБС, инфаркта миокарда и др. Неожиданный результат обнаружения более высоких значений атерогенных фракций липидов у больных с ГАЖ требует дополнительной проверки. Аостоверной корреляционной связи ИММАЖ и АПНПне получено. Возможно, роль Аислипидемии в сердечно-сосудистом континууме у бурятских пациентов не столь значима, как у русских.

\section{Заключение}

Распространенность ГАЖ среди бурятских пациентов с АГ составляет $62 \pm 4,9 \%$. Выявляется тенденция к более высокой частоте ГАЖ у женщин $(69,8 \pm 6,3 \%)$, чем у мужчин $(53,2 \pm 7,3 \%)$. У женщин существенно чаще встречается значительная ГАЖ. Наиболее частым типом ремоделирования $\Lambda Ж$ у бурятских пациентов является концентрическая ГАЖ, которая также значимо чаще обнаруживается у женщин $(47,2 \pm 6,9 \%)$, чем у мужчин $(34 \pm 6,9 \%)$. 


\section{Аитература}

1. Абрамов Е.А., Невзорова В.А., Настрадин О.В. Особенности проявления метаболического синдрома у женщин различной этнической принадлежности // Проблемы женского здоровья. - 2007. - № 1. - С. 20-29.

2. Аонская А.А., Морозов С.Н., Морозова Е.А. Артериальная гипертония на Севере// Якутский медицинский журнал. - 2008. - № 2 (22). - С. 22-25.

3. Рекомендации по количественной оценке структуры и функции камер сердца: пер. с англ. // Российский кардиологический журнал. - 2012. - № 3 (95). - С. 1-28.

4. Bella J.N., Goring H.H. Genetic epidemiology of left ventricular hypertrophy // Am. J. Cardiovasc. Dis. - 2012. Vol. 2, № 14. - P. 267-278.

5. Chantra S., Bhutong B. Echocardiographically detected left ventricular hypertrophy: prevalence and risk factors in Thai elderly men and women // J. Ned. Assoc. Thai. 2008. Vol. 83, № 9. - P. 1082-1094.

6. Cuspidi C., Sala C., Negri F., Mancia A., Morganti A. Prevalence of left-ventricular hypertrophy in hypertension: an updated review of echocardiographic studies // J. Hum. Hypertension. - 2012. - Vol. 26, № 6. - P. 343-349.

7. Devereux R.B., Alonso D.R., Lutas E.M. Gottlieb G.J., Campo E., Sachs J., Reichek N. Echocardiographic assessment of left ventricular hypertrophy: comparison to necropsy findings // Am. J. Cardiol. - 1986. - Vol. 57. P. 450-458.

8. Kannel $\mathrm{Wb}$, Cobb J. Left ventricular hypertrophy and mortality- results from the Framingham Study // Cardiology. - 1992. - Vol. 81, №4-5. - P. 291-298.

9. Koren M.J., Devereux R.B., Casale P.N., Savage D.D., Laraqh J.H. Relation of left ventricular mass and geometry to morbidity and mortality in uncomplicated essential hypertension // Ann. Intern. Med. - 1991. - Vol. 114. P. $345-352$.

10. Krumholz H.M., Larson M., Levy D. Prognosis of left vernacular geometric patterns in Framingham Heart Study // J .Am. Coll. Cardiol. - 1995. - Vol. 25, № 4. -P. 879-884.

11. Park C.M., March K., Ghosh A.K., Jones S., Coady E., Tuson C., Francis D., Mayet J., Tillin T., Chaturvedi N., Hughes A.D. Left-Ventricular Structure in the Southall And Brent REvisited (SABRE) Study: Explaining Ethnic Differences. Hypertension. - 2013. - Vol. 61, № 5. - P. 1014-1020.

12. Wang S.X., Xue H., Zou Y.B., Sun K., Fu C.Y., Wang H., Hui R.T. Prevalence and risk factors for left ventricular geometric abnormality in patients with hypertension among Han Chinese // Chin. Med. J. - 2012. - Vol. 125, № 1. P. 21-26.

\section{References}

1. Abramov E.A., Nevzorova V.A., Nastradin O.V. Features of the metabolic syndrome in women of different ethnic backgrounds // Problems of Women's Health. - 2007. № 1. - P.20-29.

2. Donskaya A.A., Morozov S.N., Morozova E.A. Hypertension in the North //Yakut Medical Journal. - 2008. № 2 (22). - P. 22-25.
3. Recommendations for quantitative evaluation of the structure and function of the heart chambers: Transl. from English // Russian Journal of Cardiology. - 2012. - № 3 (95). - P. 1-28.

4. Bella J.N., Goring H.H. Genetic epidemiology of left ventricular hypertrophy // Am. J. Cardiovasc. Dis. 2012. - Vol. 2, № 14. - P. 267-278.

5. Chantra S., Bhutong B. Echocardiographically detected left ventricular hypertrophy: prevalence and risk factors in Thai elderly men and women // J. Ned. Assoc. Thai. 2008. - Vol. 83, № 9. - P. 1082-1094.

6. Cuspidi C., Sala C., Negri F., Mancia A., Morganti A. Prevalence of left-ventricular hypertrophy in hypertension: an updated review of echocardiographic studies // J. Hum. Hypertension. - 2012. - Vol. 26, № 6. - P. 343-349.

7. Devereux R.B., Alonso D.R., Lutas E.M. Gottlieb G.J., Campo E., Sachs J., Reichek N. Echocardiographic assessment of left ventricular hypertrophy: comparison to necropsy findings // Am. J. Cardiol. - 1986. - Vol. 57. - P. 450-458.

8. Kannel $\mathrm{Wb}$, Cobb J. Left ventricular hypertrophy and mortality- results from the Framingham Study // Cardio-logy. - 1992. - Vol. 81, № 4-5. - P. 291-298.

9. Koren M.J., Devereux R.B., Casale P.N., Savage D.D., Laraqh J.H. Relation of left ventricular mass and geometry to morbidity and mortality in uncomplicated essential hypertension // Ann. Intern. Med. - 1991. - Vol. 114. P. 345-352.

10. Krumholz H.M., Larson M., Levy D. Prognosis of left vernacular geometric patterns in Framingham Heart Study // J .Am. Coll. Cardiol. - 1995. - Vol. 25, № 4. -P. 879-884.

11. Park C.M., March K., Ghosh A.K., Jones S., Coady E., Tuson C., Francis D., Mayet J., Tillin T., Chaturvedi N., Hughes A.D. Left-Ventricular Structure in the Southall And Brent REvisited (SABRE) Study: Explaining Ethnic Differences. Hypertension. - 2013. - Vol. 61, № 5. - P. 1014-1020.

12. Wang S.X., Xue H., Zou Y.B., Sun K., Fu C.Y., Wang H., Hui R.T. Prevalence and risk factors for left ventricular geometric abnormality in patients with hypertension among Han Chinese // Chin. Med. J. - 2012. - Vol. 125, № 1. P. 21-26.

\section{Сведения об авторах}

Марханова Елена Сергеевна - аспирант кафеgры госпитальной терапии ГБОУ ВПО Иркутский госуяарственный меgицинский университет МЗ РФ.

Agрес: 664003, г. Иркутск, ул. Красного Восстания g. 1; тел. 8(93952) 407920; e-mail: emarkhanova@mail.ru.

Орлова Галина Михайловна - gоктор меgицинских наук, профессор, завеgующая кафеgрой госпитальной терапии, ГБОУ ВПО Иркутский государственный медищинский университет МЗ РФ.

Agpec: 664003, г. Иркутск, ул. Красного Восстания g. 1; тел. 8(3952) 407926; e-mail: vicgal@yandex.ru.

\section{Authors}

Markhanova Elena Sergeevna - Postgraduate Student, Department of Hospital Therapy, Irkutsk State Medical University, Ministry of Health of the Russian Federation. Address: 1, Krasnogo Vosstania Str., Irkutsk, RF, 664003; Phone: 8(908) 6569670, e-mail: emarkhanova@mail.ru.

Orlova Galina Mikhailovna-Dr.Med.Sc., Professor, the Head of the Department of Hospital Therapy, Irkutsk State Medical University, Ministry of Health of the Russian Federation

Address: 1, Krasnogo Vosstania Str., Irkutsk, RF, 664003; Phone: 8 (3952) 407926, e-mail: vicgal@yandex.ru. 